Copyright by the American Institute of Physics (AIP). Ge, Wenwei; Maurya, Deepam; Li, Jiefang; et al., "Alternating and direct current field effects on the structure-property relationships in Na0.5Bi0.5TiO3-x० BaTiO3 textured ceramics," Appl. Phys. Lett. 102, 222905 (2013); http://dx.doi.org/10.1063/1.4809940

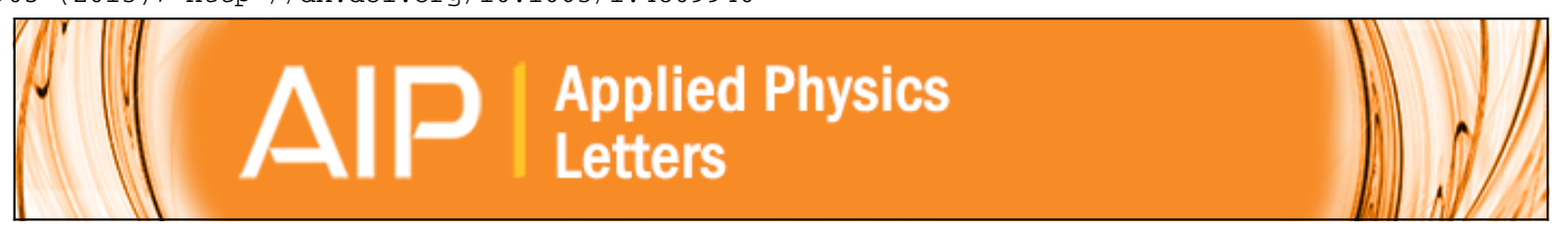

Alternating and direct current field effects on the structure-property relationships in Na0.5Bi0.5TiO3-x\%BaTiO3 textured ceramics

Wenwei Ge, Deepam Maurya, Jiefang Li, Shashank Priya, and D. Viehland

Citation: Applied Physics Letters 102, 222905 (2013); doi: 10.1063/1.4809940

View online: http://dx.doi.org/10.1063/1.4809940

View Table of Contents: http://scitation.aip.org/content/aip/journal/apl/102/22?ver=pdfcov

Published by the AIP Publishing

AlP Re-register for Table of Content Alerts 


\title{
Alternating and direct current field effects on the structure-property relationships in $\mathrm{Na}_{0.5} \mathrm{Bi}_{0.5} \mathrm{TiO}_{3}-\mathrm{X}_{\mathrm{BaTiO}}$ textured ceramics
}

\author{
Wenwei Ge, ${ }^{1, a)}$ Deepam Maurya ${ }^{2}$ Jiefang Li, ${ }^{1}$ Shashank Priya, ${ }^{2}$ and D. Viehland ${ }^{1}$ \\ ${ }^{1}$ Department of Materials Science and Engineering, Virginia Tech, Blacksburg, Virginia 24061, USA \\ ${ }^{2}$ Center for Energy Harvesting Materials and Systems (CEHMS), Bio-Inspired Materials and Devices \\ Laboratory (BMDL), Virginia Tech, Blacksburg, Virginia 24061, USA
}

(Received 23 April 2013; accepted 25 May 2013; published online 6 June 2013)

\begin{abstract}
The influence of alternating (ac) and direct current (dc) fields on the structural and dielectric properties of $[001]_{\mathrm{PC}}$ textured $\mathrm{Na}_{0.5} \mathrm{Bi}_{0.5} \mathrm{TiO}_{3}-7 \% \mathrm{BaTiO}_{3}(\mathrm{NBT}-7 \% \mathrm{BT})$ ceramics has been investigated. X-ray diffraction measurements revealed that the depolarization at temperature $T_{d}$ in poled samples resulted from a tetragonal $\rightarrow$ pseudo-cubic transition on heating. Moderate ac drive and dc bias had opposite influences on $T_{d}$ : ac drive decreased the $T_{d}$, whereas dc bias increased it. These investigations suggested an effective method to expand the working temperature range of NBT- $x \%$ BT textured ceramics to a high temperature. (C) 2013 AIP Publishing LLC.

[http://dx.doi.org/10.1063/1.4809940]
\end{abstract}

Solid solutions of $\mathrm{Na}_{0.5} \mathrm{Bi}_{0.5} \mathrm{TiO}_{3}-x \% \mathrm{BaTiO}_{3}$ (abbreviated as NBT- $x \%$ BT) are potentially important lead-free piezoelectric systems, as they have a rhombohedral (R) to tetragonal $(\mathrm{T})$ phase transition at a morphotropic phase boundary (or MPB) near $6<x<8 .{ }^{1,2}$ At this MPB, weakfield $\mathrm{d}_{33}$ values as high as $480 \mathrm{pC} / \mathrm{N}$ have been reported in Mn-doped NBT-5.6\% BT single crystals, ${ }^{3}$ via a Berlincourt method.

On cooling, the dielectric constant of NBT- $x \% \mathrm{BT}$ first shows a broad but frequency independent maximum at a temperature $T_{\max }$, and then exhibits a strongly frequency dependent anomaly at a lower temperature $\mathrm{T}_{\text {hump. }}{ }^{4,5}$ In spite of the presence of peaks in the dielectric response, $x$-ray diffraction has not revealed any apparent non-cubic distortions near the MPB at any temperature in the absence of an applied electric field (E). ${ }^{6-8}$ Transmission electron microscopy (TEM) studies have demonstrated the coexistence of nano-scale regions with $R 3 \mathrm{c}$ and P4bm symmetries within an average cubic matrix for compositions near and below the MPB., ${ }^{4,9,10}$

$\mathrm{X}$-ray diffraction has revealed a long range ordered lower symmetry (i.e., ferroelectric) structure for poled NBT$x \% \mathrm{BT}$ that was dependent on the crystallographic direction along which E-field was applied. Average $\mathrm{T}$ and $\mathrm{R}$ phases were induced by applying dc E-field $\left(\mathrm{E}_{\mathrm{dc}}\right)$ along [001] and [111], respectively. ${ }^{11}$ The E-field induced pseudo-cubic $\rightarrow \mathrm{T}$ transformation along [001] yields a strain as high as $\varepsilon=0.6 \%$ under $\mathrm{E}_{\mathrm{dc}}=20 \mathrm{kV} / \mathrm{cm},{ }^{11,12}$ which is 3 times larger than the typical strains of lead zirconate titanate (PZT) ceramics. ${ }^{18}$ In order to take full advantage of this high strain in polycrystalline materials, [001] textured NBT- $x \%$ BT ceramics have recently been developed. ${ }^{13}$ An E-field induced transformation from a long range ferroelectric state to a relaxor-like condition is known on zero field heating the poled sample at a previously so-called depolarization temperature or $\mathrm{T}_{\mathrm{d}},{ }^{4,5}$ at which point the longitudinal piezoelectric coefficient also goes to zero. This temperature $T_{d}$ is frequency independent and hundreds of degrees below $T_{\max }$. Please note that the $T_{\text {hump }}$ is used for

${ }^{\text {a)} E l e c t r o n i c ~ m a i l: ~ w e n w e i g e @ v t . e d u ~}$ unpoled samples and represents the temperature where the frequency dispersion in the dielectric spectrum is reduced, while $T_{d}$ is used for poled samples where the piezoelectric properties disappear. The temperature values are $\mathrm{T}_{\mathrm{d}}<\mathrm{T}_{\text {hump }}<\mathrm{T}_{\max }$. The dielectric relaxation and a square-to-slim P-E loop transition with increasing temperature below $\mathrm{T}_{\text {hump }}$ are similar to that of $\mathrm{Pb}$-based perovskite relaxors, such as $\left(\mathrm{Pb}_{1-3 x / 2} \mathrm{La}_{x}\right)\left(\mathrm{Zr}_{y} \mathrm{Ti}_{1-y}\right) \mathrm{O}_{3}$ or PLZT100 $x / 100 y / 100(1-y)$ with $\mathrm{x}=0.08$ and $\mathrm{y}=0.65 .^{4}$ Thus, it has been proposed that NBT- $x \% \mathrm{BT}$ is a relaxor ferroelectric, which has a coexistence of polar nanoregions of $R 3 c$ and $P 4 \mathrm{bm}$ symmetries. ${ }^{4,14}$ The two dielectric anomalies for NBT- $x \% \mathrm{BT}$ at $\mathrm{T}_{\text {hump }}$ and $\mathrm{T}_{\max }$ were then attributed to the thermal evolution of these two types of polar nanoregions. ${ }^{4}$

Previous investigations of PLZT $x / 65 / 35$ have also shown that a relaxor state can be induced from a long-range ferroelectric state with an increasing ac E-field $\left(\mathrm{E}_{\mathrm{ac}}\right)$. For $x=0.02$ $\sim 0.06$, dielectric measurements revealed a frequency dependent dielectric response for $\mathrm{E}_{\mathrm{ac}} \approx 500 \mathrm{~V} / \mathrm{cm}$ that was similar to that of a relaxor ferroelectric; whereas at lower $E_{a c}$, the dielectric response was diffuse like a "soft" ferroelectric but dispersionless. ${ }^{15}$ Recently, it was reported that $T_{d}$ of poled Mn-doped NBT- $x \%$ BT was shifted towards higher temperature with increasing $\mathrm{E}_{\mathrm{dc}}$, due to the stabilization of polarization. ${ }^{16}$ The fatigue behavior of NBT-6\%BT under bipolar Efield has previously indicated that the ferroelectric domain structure may become fragmented. ${ }^{17}$ However, previous dielectric studies of NBT- $x \%$ BT have been performed using relatively small $\mathrm{E}_{\mathrm{ac}}$ drives. The purpose of this study was to perform a detailed investigation of the dielectric response and structural changes under moderate $\mathrm{E}_{\mathrm{ac}}$ and $\mathrm{E}_{\mathrm{dc}}$ for poled and unpoled NBT- $x \%$ BT textured ceramics near the MPB. Our findings showed that the $T_{d}$ can be increased by $40^{\circ} \mathrm{C}$ under a moderate $\mathrm{E}_{\mathrm{dc}}$ of $12 \mathrm{kV} / \mathrm{cm}$ in the NBT-7\% BT textured ceramics.

[001] textured NBT-7\%BT ceramics were prepared using a tape casting technique. ${ }^{18}$ The samples were then sintered in air at 1150 to $1200^{\circ} \mathrm{C} .{ }^{13}$ The degree of [001] texture, as calculated by the Lotgering factor method, ${ }^{19}$ was about $\sim 92 \%$. NBT-7\%BT textured ceramic samples of dimensions 
$2 \times 2 \times 0.42 \mathrm{~mm}^{3}$ were cut, polished, and subsequently electroded on both surfaces with gold. The samples were poled along the textured direction under $\mathrm{dc} E=26 \mathrm{kV} / \mathrm{cm}$ for $15 \mathrm{~min}$ at room temperature. Dielectric measurements were performed using a LCR meter (HP $4284 \mathrm{~A}$ ) over a frequency range from 20 to $10^{6} \mathrm{~Hz}$ and under an ac drive in the range between 0.005 and $20 \mathrm{~V}$. Temperature dependent dielectric measurements were made by placing samples in a Delta Design 9023 test chamber. A dc electrical bias field was applied through a blocking circuit, which could be adjusted between 0 and $2000 \mathrm{~V}$. All measurements were performed upon heating. Temperature dependent (200) peaks were taken under various dc biases between 0 and $12 \mathrm{kV} / \mathrm{cm}$. These studies were done using a Philips MPD high-resolution X-ray diffraction system, equipped with a two bounce hybrid monochromator, an open three-circle Eulerian cradle, and a domed hot-stage. A Ge (220)-cut crystal was used as an analyzer, which had an angular resolution of $0.0068^{\circ}$. The X-ray wavelength was that of $\mathrm{Cu} K_{\alpha}=1.5406 \AA$.

Figures 1(a) and 1(b) show the temperature dependent dielectric constant $\varepsilon_{\mathrm{r}}$ and loss factor $\tan \delta$ for unpoled and poled [001]-textured NBT-7\%BT ceramics. These data were taken on heating. The results reveal a diffuse phase transformation with a broad dielectric maximum near $\mathrm{T}_{\max }=290^{\circ} \mathrm{C}$ (see inset of Fig. 1(a)). In the unpoled state, the dielectric constant was strongly frequency dependent below $\mathrm{T}_{\text {hump }}=160^{\circ} \mathrm{C}$, and only weakly frequency dependent between $160^{\circ} \mathrm{C}$ and $290^{\circ} \mathrm{C}$. The dielectric anomaly near $160^{\circ} \mathrm{C}$ has been attributed to the thermal evolution of polar nano-regions from $\mathrm{R} 3 \mathrm{c}$ to $\mathrm{P} 4 \mathrm{bm}$ symmetries. ${ }^{4}$ In the poled condition, the dielectric constant was found to be decreased and nearly frequency independent below $\mathrm{T}_{\mathrm{d}}=80^{\circ} \mathrm{C}$, but abruptly became strongly frequency dependent on heating above $\mathrm{T}_{\mathrm{d}}$. It remained strongly frequency dependent on heating to $160{ }^{\circ} \mathrm{C}$, and then subsequent became less dispersive on further heating above $160{ }^{\circ} \mathrm{C}$. Comparisons of the temperature dependent dielectric constant for the unpoled and poled conditions shows that a normal long range ordered ferroelectric state exists in the poled state, which may undergo a normal to relaxor transition on heating near $\mathrm{T}_{\mathrm{d}}=80^{\circ} \mathrm{C}$. A sharp increase of $\tan \delta$ was also found at $\mathrm{T}_{\mathrm{d}}=80^{\circ} \mathrm{C}$ on heating for poled samples, and remained high over the temperature range of 80 to $160^{\circ} \mathrm{C}$.

The dielectric response for the unpoled and poled [001]-textured NBT-7\%BT ceramics as a function of temperature at various frequencies between $10^{2}$ and $10^{5} \mathrm{~Hz}$ was investigated using drive amplitudes of 10,100 , and $500 \mathrm{~V} / \mathrm{cm}$, respectively. For the unpoled condition, the dielectric response showed the same strong frequency dispersion below $\mathrm{T}_{\text {hump }}=160{ }^{\circ} \mathrm{C}$ in both the low- and highamplitude responses (data not shown). Increasing ac drive had some effects on the dielectric response as shown in the Figure 2(a). The magnitude of the dielectric constant at $1 \mathrm{kHz}$ was slightly increased above $160^{\circ} \mathrm{C}$, however the nonlinearities were weak in the ac drive amplitude range investigated. For the poled conditions, the influence of ac drive amplitudes on the nonlinearities and frequency dispersion of the dielectric response were also weak, however the $\mathrm{T}_{\mathrm{d}}$ temperature was decreased from $80^{\circ} \mathrm{C}$ to $75^{\circ} \mathrm{C}$ with
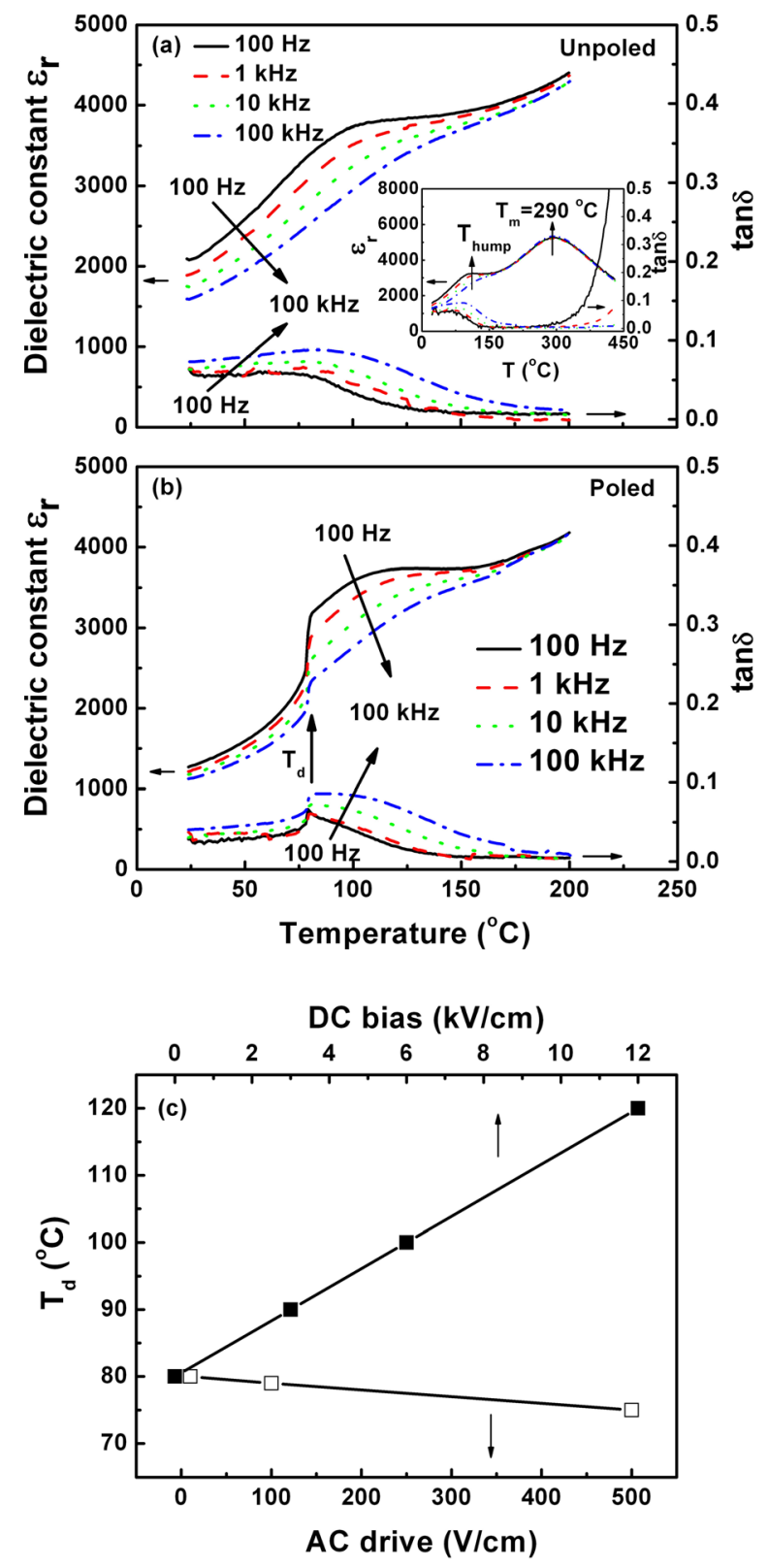

FIG. 1. Temperature dependence of the dielectric constant $\varepsilon_{\mathrm{r}}$ and loss factor $\tan \delta$ for (a) unpoled and (b) poled [001]-textured NBT-7\%BT ceramics measured at frequencies of $100 \mathrm{~Hz}, 1 \mathrm{kHz}, 10 \mathrm{kHz}$, and $100 \mathrm{kHz}$ using AC drive of $10 \mathrm{~V} / \mathrm{cm}$. The dielectric maximum of [001]-textured NBT-7\%BT ceramics occurred at temperature of $290^{\circ} \mathrm{C}$, as indicated by the inset of (a). The influence of DC bias and AC drive on the depolarization temperature $T_{d}$ of poled samples were summarized in (c). The solid and open symbols represent the value of $\mathrm{T}_{\mathrm{d}}$ as functions of dc bias and ac drive amplitude.

increasing ac drive amplitudes from $10 \mathrm{~V} / \mathrm{cm}$ to $500 \mathrm{~V} / \mathrm{cm}$ as shown in the Figure 2(b). This behavior is in line with previous results that revealed shifting of the dielectric permittivity characteristics of NBT towards lower temperatures with the increasing a.c. electric field strength. ${ }^{20}$ This phenomenon was attributed to the reactivation and reversal of polar regions under the external field.

The influence of DC bias on the dielectric response for the unpoled and poled NBT-7\%BT is shown in the Figures 3(a)-3(d). All these data were measured using $\mathrm{E}_{\mathrm{ac}}=100 \mathrm{~V} / \mathrm{cm}$. For unpoled samples, it can be seen that the $\mathrm{T}_{\text {hump }}$ of $\varepsilon_{\mathrm{r}}$ (or $\tan \delta$ ), which has been attributed to the thermal evolution of polar nano-regions from $R 3 c$ to $P 4 b m$ symmetries on heating, ${ }^{4}$ 

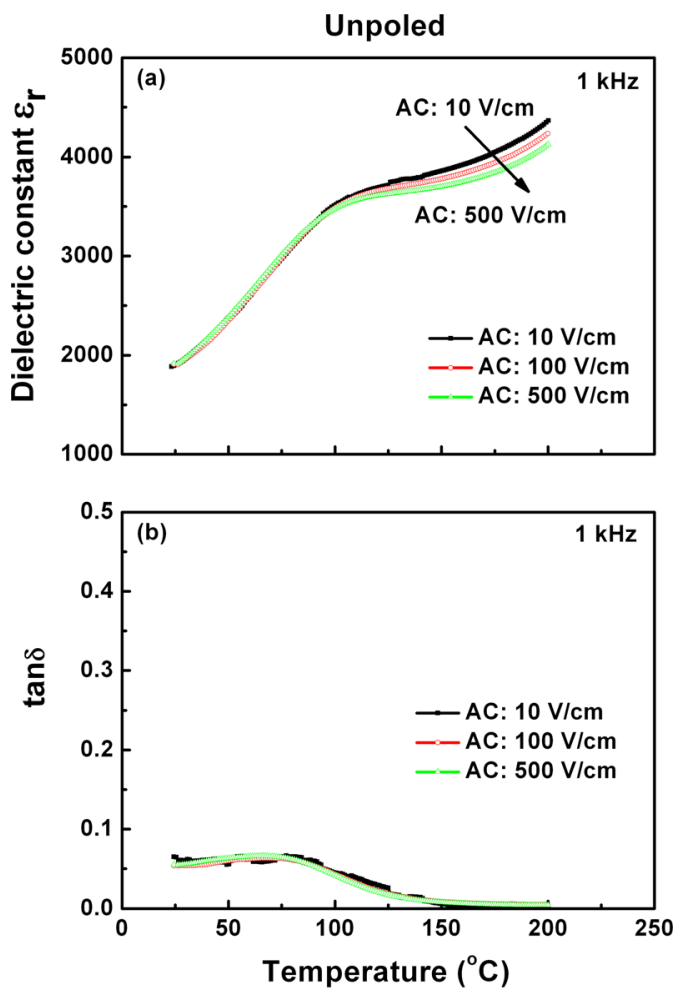

Poled
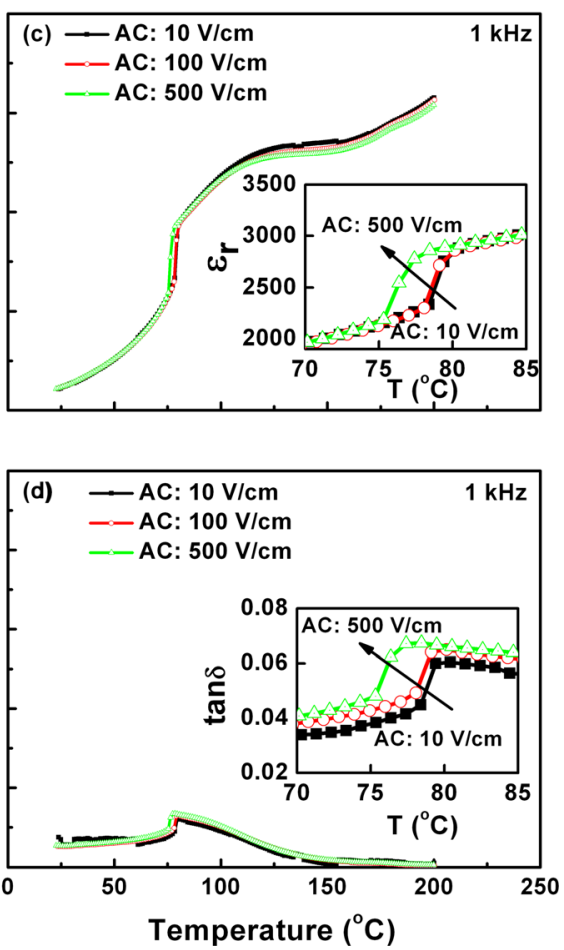

FIG. 2. Dependence of the $1 \mathrm{kHz}$ dielectric constant $\varepsilon_{\mathrm{r}}$ and loss factor $\tan \delta$ for unpoled ((a) and (b)) and poled ((c) and (d)) [001]-textured NBT-7\%BT ceramics on the amplitude of ac drive between $10 \mathrm{~V} / \mathrm{cm}$ and $500 \mathrm{~V} / \mathrm{cm}$. was increased from $100^{\circ} \mathrm{C}$ (or $65^{\circ} \mathrm{C}$ ) to $135^{\circ} \mathrm{C}$ (or $100{ }^{\circ} \mathrm{C}$ ) with increasing $\mathrm{E}_{\mathrm{dc}}$ from 0 to $12 \mathrm{kV} / \mathrm{cm}$ (see Figs. 3(a) and $3(\mathrm{~b})$ ). These results indicate that application of dc bias favors the stabilization of polar nanoregions with $R 3 c$ symmetry and increases the thermal evolution temperature range for its transformation into polar nanoregions of $P 4 \mathrm{bm}$ symmetry. ${ }^{4,14}$ Textured specimens with enhanced piezoelectric responses were reported to have polar nanoregions that have a coherence length smaller than that of randomly oriented ceramic. ${ }^{13}$ Moreover, the textured specimen with smaller and more ordered nano domains was found to show a coherent switching of domains with smaller coercive field. ${ }^{13}$ Rietveld fits to the synchrotron diffraction patterns revealed a high degree of texturing, resulting in extra structural distortions in textured NBT$7 \% \mathrm{BT}$ and changes in the polarization-electric field behavior. ${ }^{18}$ For poled samples, the application of $\mathrm{E}_{\mathrm{dc}}$ along the poling direction during heating clearly shifted the depolarization temperature $\mathrm{T}_{\mathrm{d}}$ to higher temperatures (see Figs. 3(c) and 3(d)). The value of $\mathrm{T}_{\mathrm{d}}$ was increased from $80^{\circ} \mathrm{C}$ to $100^{\circ} \mathrm{C}$ and $120^{\circ} \mathrm{C}$ with increasing $\mathrm{E}_{\mathrm{dc}}$ from $0 \mathrm{kV} / \mathrm{cm}$ to $6 \mathrm{kV} / \mathrm{cm}$ and
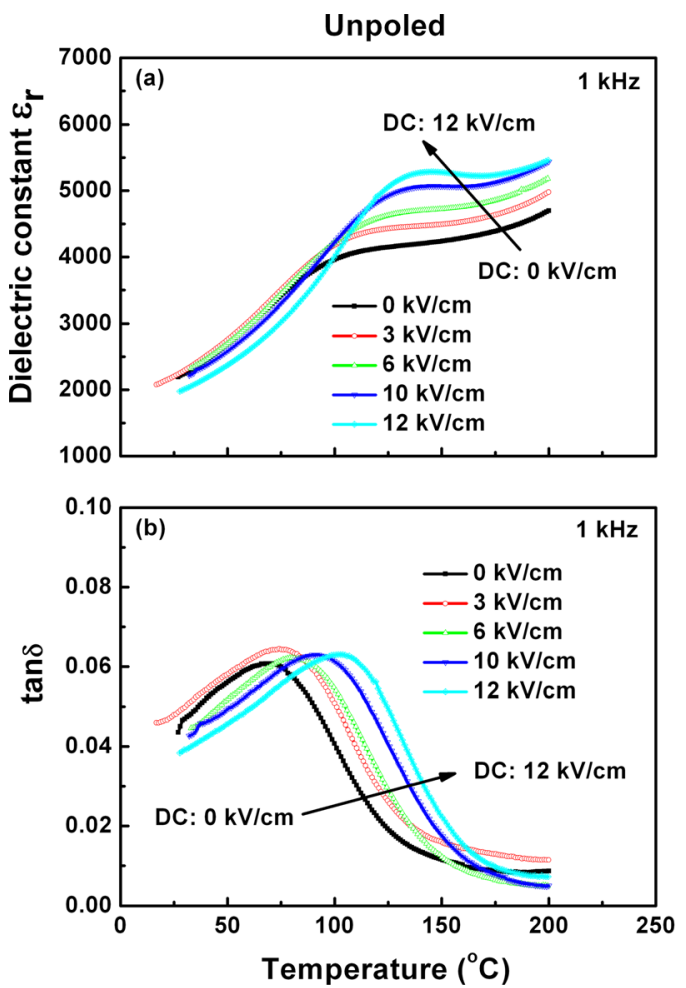
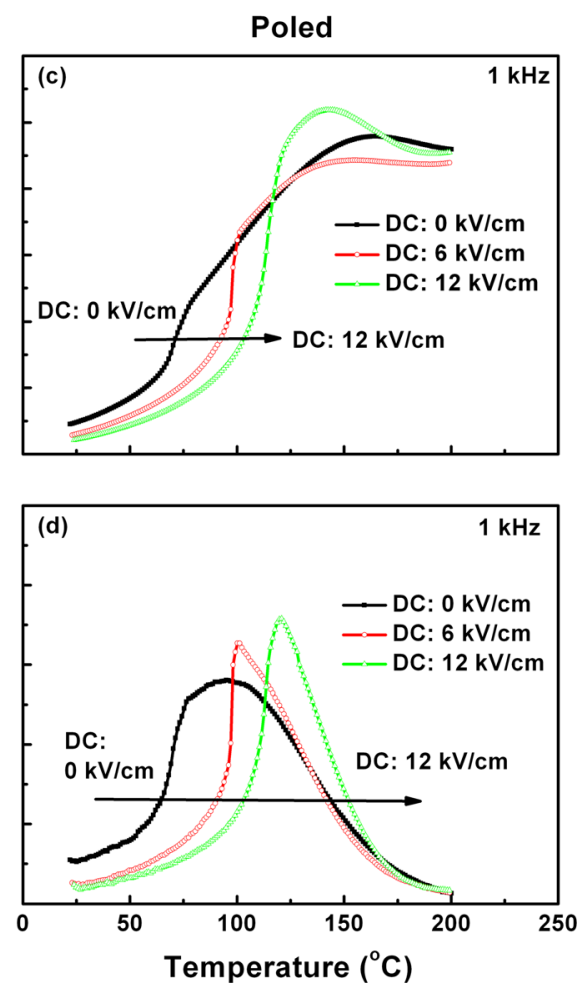

FIG. 3. Dependence of the $1 \mathrm{kHz}$ dielectric constant $\varepsilon_{\mathrm{r}}$ and loss factor $\tan \delta$ for unpoled ((a) and (b)) and poled ((c) and (d)) [001]-textured NBT-7\%BT ceramics on a DC bias between 0 and $12 \mathrm{kV} / \mathrm{cm}$. 

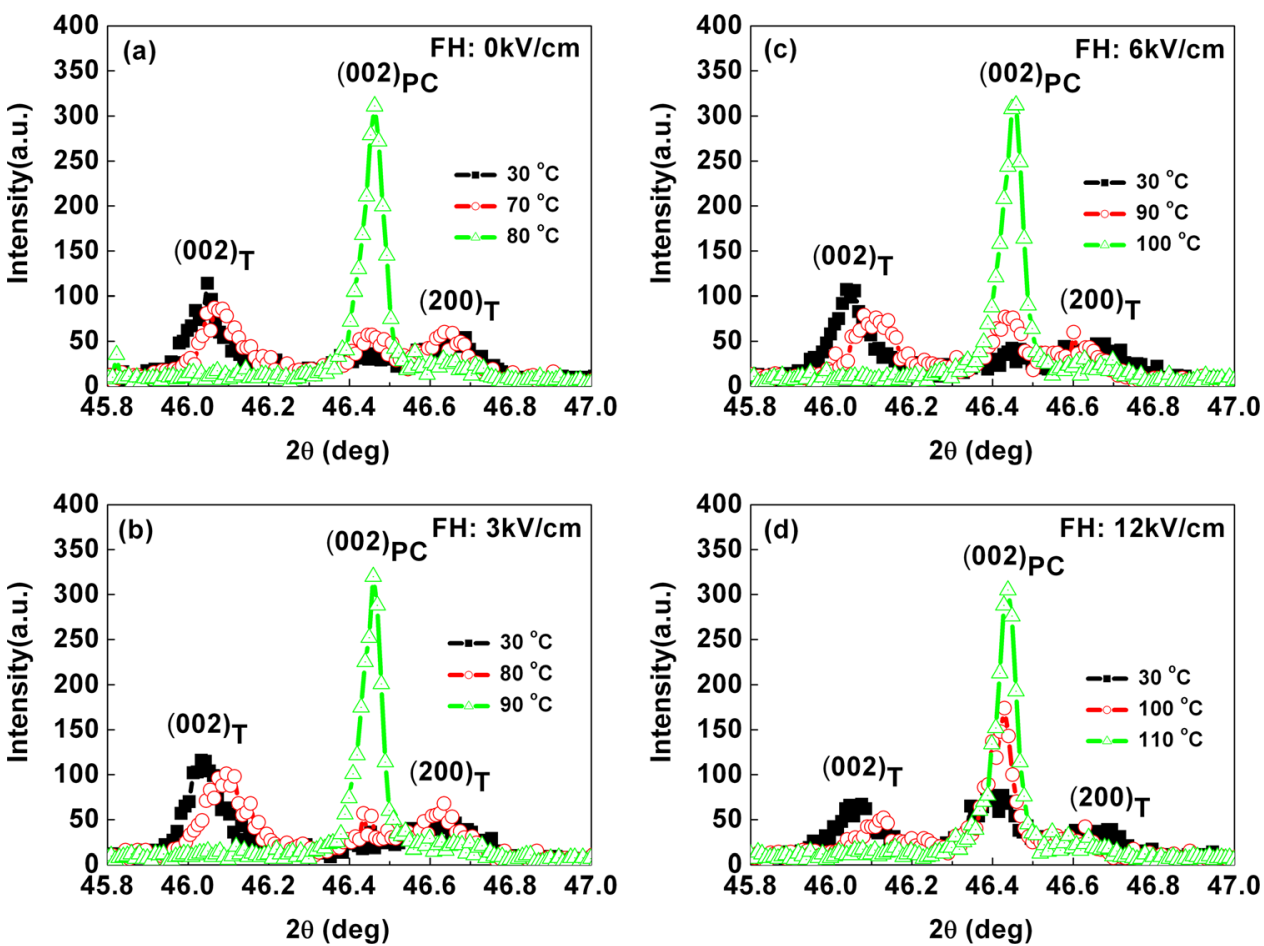

FIG. 4. Dependence of the (200) x-ray diffraction peaks for poled [001]-textured NBT-7\%BT ceramics measured under DC biases of (a) $0 \mathrm{kV} / \mathrm{cm}$, (b) $3 \mathrm{kV} / \mathrm{cm}$, (c) $6 \mathrm{kV} / \mathrm{cm}$, and (d) $12 \mathrm{kV} / \mathrm{cm}$ at temperatures between $30^{\circ} \mathrm{C}$ and $110^{\circ} \mathrm{C}$.
$12 \mathrm{kV} / \mathrm{cm}$. The degree of the shift for $T_{d}$ in the [001]-textured ceramics was $2 \times$ of that in random-oriented ceramic samples. ${ }^{16}$ These results indicate that texture is an effective method to shift the $\mathrm{T}_{\mathrm{d}}$ to higher temperature on application $\mathrm{E}_{\mathrm{dc}}$ to NBT-x\%BT ceramics exhibiting a practical relevance of textured specimen.

To understand the structural origin of the influence of $\mathrm{E}_{\mathrm{dc}}$ on the properties of [001]-textured NBT-7\%BT ceramics, the temperature evolution of the (200) peak for poled samples was measured on heating under $0 \mathrm{kV} / \mathrm{cm} \leq \mathrm{E}_{\mathrm{dc}} \leq 12 \mathrm{kV} / \mathrm{cm}$ by $\mathrm{x}$-ray diffraction. Figure 4(a) shows the (200) peak for the poled sample at three selected temperatures under zero-field conditions. X-ray powder diffraction revealed that the unpoled [001]-textured NBT-7\%BT ceramics had an average cubic (or pseudo-cubic) structure. This result is consistent with that reported in previous studies. ${ }^{4,13}$ After poling under $\mathrm{E}_{\mathrm{dc}}=26 \mathrm{kV} / \mathrm{cm}$, the (200) peak spit into three peaks centered at $2 \theta=46.05^{\circ}, 46.46^{\circ}$, and $46.68^{\circ}$, as can be seen in Fig. 4(a). According to previously studies, ${ }^{7,21}$ these results indicate that an E-field induced tetragonal phase coexisted with the initial pseudo-cubic one in the poled state. The induced ferroelectric $\mathrm{T}$ phase was stable at temperatures of $\leq 70^{\circ} \mathrm{C}$, and then transformed into a pseudo-cubic one at $80^{\circ} \mathrm{C}$ on zero-field heating. The temperature of the $\mathrm{T} \rightarrow$ pseudo-cubic transition was consistent with the value of $T_{d}$ identified in the dielectric constant and loss factor data as a function of temperature (see Fig. 1(b)). These results clearly demonstrate that the depolarization of poled [001]-textured NBT-7\%BT ceramics resulted from a transition between long ranged ordered ferroelectric $\mathrm{T}$ and relaxor like pseudo-cubic phases. Application of $\mathrm{E}_{\mathrm{dc}}=3 \mathrm{kV} / \mathrm{cm}, 6 \mathrm{kV} / \mathrm{cm}$, and $12 \mathrm{kV} / \mathrm{cm}$ along the poling direction shifted the $\mathrm{T} \rightarrow$ pseudo-cubic transition to higher temperatures of $90^{\circ} \mathrm{C}, 100^{\circ} \mathrm{C}$, and $110^{\circ} \mathrm{C}$ (see Figs. 4(b)-4(d)), respectively. Note that the degree of the shift of the $\mathrm{T} \rightarrow$ pseudo-cubic transition temperature was consistent with that of the $T_{d}$ shift in the dielectric data on applying
$E_{d c}$ (see Figs. 3(c) and 3(d)). These results indicate that $T_{d}$ shifts to higher temperature in [001]-textured NBT-7\%BT ceramics by applying $\mathrm{E}_{\mathrm{dc}}$ due to the stabilization of a long range ordered ferroelectric $\mathrm{T}$ phase.

The influence of ac drive and dc bias on the dielectric properties of [001]-textured NBT-7\%BT ceramics was summarized in Fig. 1(c). The solid and open symbols represent the value of $T_{d}$ as a function of dc bias and ac drive amplitude. These results revealed that ac drive and dc bias work in opposition to each other: ac drive decreases $T_{d}$, whereas dc bias increases it. This because dc bias favors the stabilization of the long ranged ordered ferroelectric $\mathrm{T}$ state and an alignment of domains, whereas moderate ac drive favors depoling and a partial breakdown of the macro-domains. In the unpoled state, only polar nanodomains (i.e., without macrodomains) have been reported. ${ }^{9}$ Thus, moderate ac drives have no obvious influence on the dielectric properties in the unpoled condition.

In summary, the influence of ac drive and dc bias on the dielectric and structural properties of [001]-textured NBT$7 \%$ BT ceramics was investigated for poled and unpoled conditions. A relaxor like pseudo-cubic phase exists in the unpoled state. In this case, moderate ac drives have no obvious influence on the relaxor characteristic below the temperature $T_{\text {hump }}$, whereas dc bias effectively shifts $T_{\text {hump }}$ to higher temperatures due to the stabilization of polar nanoregions with R3c symmetry. In the poled condition, a coexistence of long range ordered ferroelectric $\mathrm{T}$ and pseudo-cubic phases was observed. X-ray diffraction revealed that the depolarization at $\mathrm{T}_{\mathrm{d}}$ resulted from a $\mathrm{T} \rightarrow$ pseudo-cubic transition on heating. Moderate ac drive and dc bias have opposite influence on the $T_{d}$ : ac drive decreases the $T_{d}$, whereas dc bias increases it.

This work was supported by the Department of Energy under DE-FG02-07ER46480. 
${ }^{1}$ T. Takenaka, K. Maruyama, and K. Sakata, Jpn. J. Appl. Phys. 30(9B), 2236 (1991).

${ }^{2}$ W. Jo, J. E. Daniels, J. L. Jones, X. Tan, P. A. Thomas, D. Damjanovic, and J. Rödel, J. Appl. Phys. 109(1), 014110 (2011).

${ }^{3}$ Q. H. Zhang, Y. Y. Zhang, F. F. Wang, Y. J. Wang, D. Lin, X. Y. Zhao, H. S. Luo, W. W. Ge, and D. Viehland, Appl. Phys. Lett. 95(10), 102904 (2009).

${ }^{4}$ W. Jo, S. Schaab, E. Sapper, L. A. Schmitt, H.-J. Kleebe, A. J. Bell, and J. Rödel, J. Appl. Phys. 110(7), 074106 (2011).

${ }^{5}$ B. Wylie-van Eerd, D. Damjanovic, N. Klein, N. Setter, and J. Trodahl, Phys. Rev. B 82(10), 104112 (2010).

${ }^{6}$ W. Ge, H. Cao, C. DeVreugd, J. Li, D. Viehland, Q. Zhang, and H. Luo, J. Am. Ceram. Soc. 94(9), 3084 (2011).

${ }^{7}$ J. E. Daniels, W. Jo, J. Rodel, and J. L. Jones, Appl. Phys. Lett. 95(3), 032904 (2009).

${ }^{8}$ J. E. Daniels, W. Jo, J. Rodel, V. Honkimaki, and J. L. Jones, Acta Mater. 58(6), 2103 (2010).

${ }^{9}$ J. Yao, N. Monsegue, M. Murayama, W. Leng, W. T. Reynolds, Q. Zhang, H. Luo, J. Li, W. Ge, and D. Viehland, Appl. Phys. Lett. 100(1), 012901 (2012).

${ }^{10}$ J. Kling, X. L. Tan, W. Jo, H. J. Kleebe, H. Fuess, and J. Rodel, J. Am. Ceram. Soc. 93(9), 2452 (2010).
${ }^{11}$ C. Luo, W. Ge, Q. Zhang, J. Li, H. Luo, and D. Viehland, Appl. Phys. Lett. 101(14), 141912 (2012).

${ }^{12}$ W. Ge, C. Luo, Q. Zhang, C. P. Devreugd, Y. Ren, J. Li, H. Luo, and D. Viehland, J. Appl. Phys. 111(9), 093508 (2012).

${ }^{13}$ D. Maurya, A. Pramanick, K. An, and S. Priya, Appl. Phys. Lett. 100(17), 172906 (2012).

${ }^{14}$ W. Jo, R. Dittmer, M. Acosta, J. Zang, C. Groh, E. Sapper, K. Wang, and J. Rödel, J. Electroceram. 29(1), 71 (2012).

${ }^{15}$ Q. Tan and D. Viehland, Phys. Rev. B 53(21), 14103 (1996).

${ }^{16}$ E. Sapper, S. Schaab, W. Jo, T. Granzow, and J. Rodel, J. Appl. Phys. 111(1), 014105 (2012).

${ }^{17}$ H. Simons, J. Glaum, J. E. Daniels, A. J. Studer, A. Liess, J. Rodel, and M. Hoffman, J. Appl. Phys. 112(4), 044101 (2012).

${ }^{18}$ D. Maurya, Y. Zhou, Y. Yan, and S. Priya, J. Mater. Chem. C 1(11), 2102 (2013).

${ }^{19}$ G. L. Messing, S. Trolier-McKinstry, E. M. Sabolsky, C. Duran, S. Kwon, B. Brahmaroutu, P. Park, H. Yilmaz, P. W. Rehrig, K. B. Eitel, E. Suvaci, M. Seabaugh, and K. S. Oh, Crit. Rev. Solid State Mater. Sci. 29(2), 45 (2004).

${ }^{20}$ J. Suchanicz, Ferroelectrics 209, 561 (1998).

${ }^{21}$ W. Ge, Q. Zhang, Z. Wang, J. Yao, J. Li, H. Luo, and D. Viehland, Phys. Status Solidi (RRL) 5(9), 356 (2011). 